\title{
A New Approach to Mixed/Boundary Friction Prediction Using the Logistics Curve
}

Robert lan Taylor ( $\nabla$ ritaylor@uclan.ac.uk)

University of Central Lancashire https://orcid.org/0000-0002-3132-8469

lan Sherrington

University of Central Lancashire School of Engineering

\section{Research Article}

Keywords: Mixed Lubrication, Boundary Lubrication, Friction

Posted Date: January 11th, 2022

DOI: https://doi.org/10.21203/rs.3.rs-1238410/v1

License: (9) This work is licensed under a Creative Commons Attribution 4.0 International License. Read Full License 


\title{
A New Approach to Mixed/Boundary Friction Prediction Using the Logistics Curve
}

\author{
R.I. Taylor* \& I. Sherrington \\ Jost Institute for Tribotechnology \\ University of Central Lancashire \\ Preston, UK, PR1 2HE
}

*Corresponding author: ritaylor@uclan.ac.uk, ORCID: https://orcid.org/0000-0002-3132-8469

Keywords: Mixed Lubrication, Boundary Lubrication, Friction

\begin{abstract}
There is a strong focus on improving the energy efficiency of machines. Over the last 20-30 years, one way to improve energy efficiency has been to reduce lubricant viscosity. This also has the effect of leading to thinner oil films between the machine's moving surfaces and is likely to lead to increased mixed/boundary friction. Accurately predicting friction in the mixed/boundary friction regime is therefore becoming of great importance.

The work reported here suggests that commonly used asperity friction models significantly underestimate friction in the mixed/boundary friction, and a new model, based on a logistic curve fit, gives a better estimate of mixed/boundary friction, provides good agreement with experimental friction data (from Mini Traction Machine experiments), and is much more straightforward for engineers and tribologists to apply for the estimation of mixed/boundary friction losses.
\end{abstract}

\section{Introduction}

Improvements in energy efficiency are one of the most effective ways to reduce $\mathrm{CO}_{2}$ emissions from machinery. In the last 20-30 years, one trend for improving energy efficiency has been to reduce the viscosity of machine lubricants. Although this does result in lower viscous friction if the machine components operate in the hydrodynamic lubrication regime, lower viscosity lubricants will also result in thinner oil films, and so, particularly at higher loads and low speeds, there is a greater chance that machine components will enter the mixed and boundary lubrication regimes. Accurately predicting friction in the mixed and boundary lubrication regimes is, therefore, becoming of greater importance. Early contact models, based on pioneering insightful papers by Archard et al [1, 2], and further developed by Greenwood and Williamson [3] and later from Greenwood and Tripp [4], are still used widely today in the prediction of friction losses in the mixed and boundary lubrication regimes. The models accounted for the statistical distribution of asperity heights and predicted that critical parameters (such as friction and contact area) were proportional to applied load, even though asperities were distorted elastically. Since this early work, many other asperity models which could be used in prediction of mixed and boundary lubrication have been published [5-10]. However, these models are not always that straightforward for working engineers and tribologists to apply to real practical problems, since they either involve complicated non-standard mathematical functions, or they need parameters that are not always easy to estimate. 
In general, asperity contact models do not account for the complexity of modern lubricants, in particular, those lubricants that contain additives which form surface active tribo-films. Two additives are of particular importance:

(1) Anti-wear additives of which zinc dialkyl dithiophosphate, ZDDP, is a good example. (An excellent review of this additive can be found in reference [11]). ZDDP tends to form high shear surface films that can be quite thick (100-200 nm in thickness). These surface films tend to result in high friction, and the relevant surface roughness to use (for calculating the lambda ratio, $\lambda$, which is the minimum oil film thickness divided by the combined root mean square surface roughness of the contacting surfaces) can be different from that of the metal surface that the tribo-film is deposited on.

(2) Friction modifier additives - of which glycerol mono-oleate (GMO) and molybdenum disulphide $\left(\mathrm{MoS}_{2}\right)$ are two well-known examples. (A good review of friction modifiers can be found in reference [12]). These additives are thought to form molecularly thin films on the tips of asperities (which will not affect the $\lambda$ ratio, but do alter the relationship between stress and pressure at the asperity tip) resulting in lower friction values.

Recently, an insightful paper from the Imperial College tribology group [13] reported that if the friction coefficient in the mixed and boundary regime is normalized to its value at nominal zero film thickness and plotted against the $\lambda$ ratio, both ZDDP containing lubricants, and base oils without such additives, are found to fit fairly well to a universal friction curve, provided that the surface roughness of the tribo-film is included in the calculation of the $\lambda$ ratio.

In this paper, additional data points are plotted to show that the concept of a universal curve appears to be valid. A replot of the data versus $1 / \lambda$ (on a logarithmic scale) shows the classic form of a logistic curve, and this insight leads to a simple equation to evaluate mixed and boundary friction coefficients, which fit recent experimental data. Such curves are much more convenient for working tribologists and engineers to use, compared to earlier boundary and mixed friction models.

\section{2. "Universal" Curve for Mixed/Boundary Friction}

Recently, Dawczyk et al [13] have shown that, based on Mini Traction Machine (MTM) data, a "universal" normalized friction versus $\lambda$ curve applies, both for ZDDP containing lubricants, and for base oils too.

To test this hypothesis, the authors have extended the analysis by including additional friction data from other published papers $[14,15]$ with the data from the Dawczyk et al [13]. A summary plot of the various data points is shown in Figure 1.

Note that in the graph, the vertical axis is $\mathrm{X}$, which is defined by:

$$
X=\frac{f-f_{E H D}}{f_{o}-f_{E H D}}
$$

Where $f$ is the measured friction coefficient of the lubricant, $f_{E H D}$ is the limiting friction coefficient of the lubricant (as measured in the Mini Traction Machine) at high film thickness (i.e. at high speeds) and $f_{o}$ is the maximum friction coefficient (which usually occurs at the lowest speeds). Dawczyk et al 
[13] comment that $X$ is the "fraction of load supported by boundary lubrication" and report that the total friction coefficient, $f$, is given by:

$$
f=X f_{B}+(1-X) f_{H}
$$

Where $f_{B}$ is the boundary friction coefficient when the oil film thickness is zero (which in this case is $f_{0}$ ) and $f_{H}$ is the friction coefficient appropriate for hydrodynamic (or elastohydrodynamic) friction (which in this case is $f_{E H D}$ ).

The original friction data from reference [15] was not available (for Oil E) and so data from the original graph was digitized using WebPlotDigitizer (https://automeris.io/WebPlotDigitizer/).

Data on oils ZDDP containing oils Z1 and Z2 (after 60 mins and 180 minutes of rubbing respectively, in the Mini Traction Machine at $100^{\circ} \mathrm{C}$ ) were kindly supplied by the authors of reference [13], and details of these oils, and operating conditions of the Mini Traction Machine, can be found there.

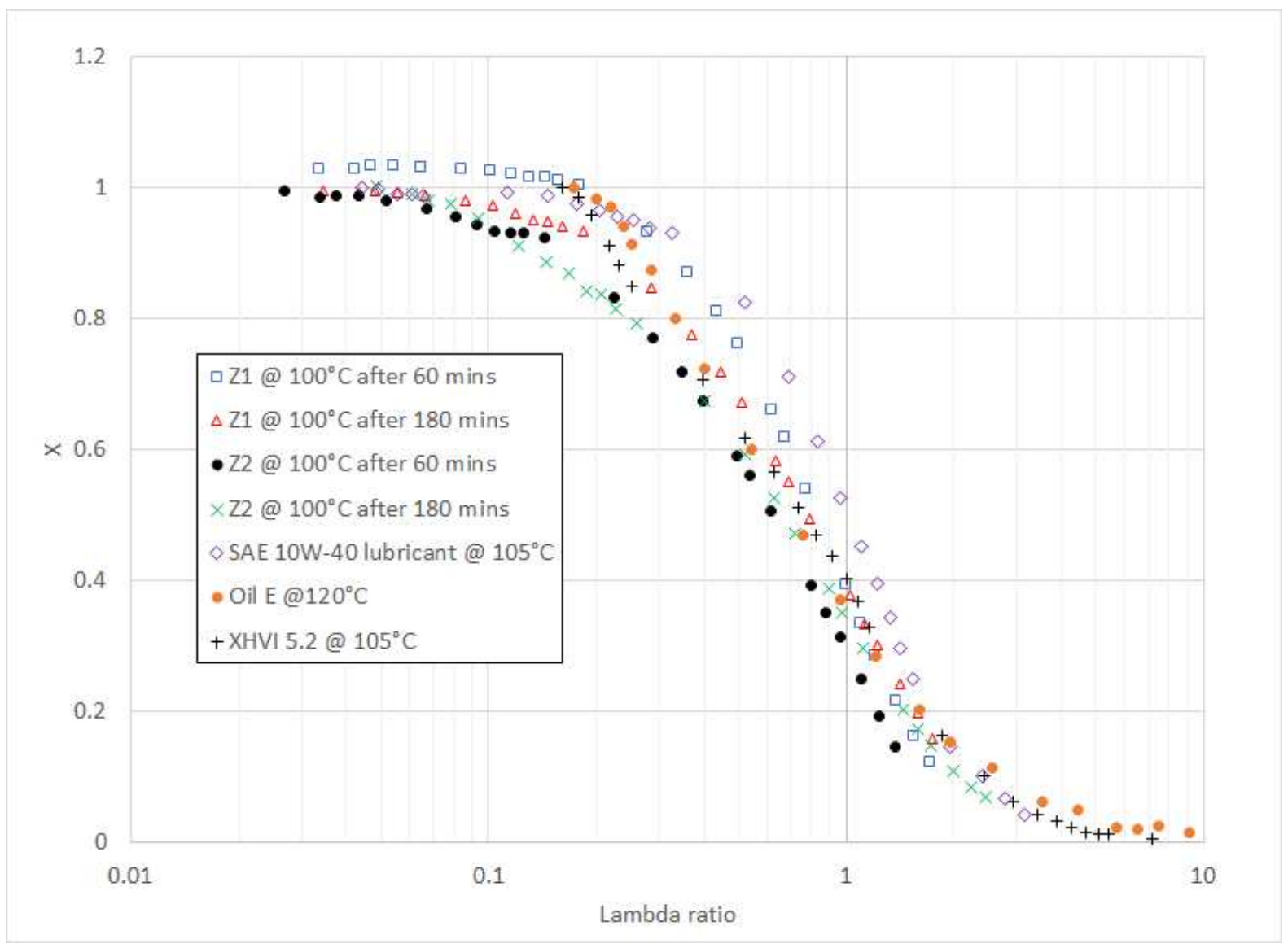

Figure 1: Graph showing "universal curve" for ZDDP containing oils (oils Z1, Z2 and the SAE 10W-40 lubricant), and base oils (oil E and XHVI 5.2), when X is plotted against $\lambda$ (data from references [13], [14] and [15])

In Figure 1, Oil E [15] is a Group 1 base oil (with $V_{k} 100=5.2 c S t$ and $V I=103$ ) and XHVI 5.2 is a Group III base oil (with $V_{k} 100=5.1 \mathrm{cSt}$ and $\mathrm{VI}=150$ ). The data on the oils $Z 1$ and $Z 2$ is from Dawczyk et al [13], and the SAE 10W-40 lubricant data is available from [14], oils studied in the latter two papers contain ZDDP anti-wear additives. The pressure viscosity coefficients for these oils (used to calculate 
the elastohydrodynamic oil film thickness in the contact) were estimated using equations from Gold et al [16].

The values of $f_{o}$ and root mean square surface roughness, $\sigma$, for the various oils in Figure 1 are summarized in Table 1 below. The larger values of $\sigma$ used for the ZDDP containing oils is because, according to Dawczyk et al [13] the tribofilms are rougher than the metal surfaces they are deposited on (at least in the case of the materials used in Mini Traction Machine tests).

\begin{tabular}{|l|l|l|}
\hline & $f_{o}$ & $\sigma(\mathrm{nm})$ \\
\hline SAE 10W-40 @ $105^{\circ} \mathrm{C}$ & 0.127 & 56.6 \\
\hline XHVI 5.2 @ $105^{\circ} \mathrm{C}$ & 0.0987 & 5.7 \\
\hline Oil E @ 120 $\mathrm{C}$ & 0.0979 & 5.7 \\
\hline Oil Z1 @ 100 $100^{\circ} \mathrm{C}(60 \mathrm{mins})$ & 0.1436 & 56.6 \\
\hline Oil Z1 @ $100^{\circ} \mathrm{C}(180 \mathrm{mins})$ & 0.1394 & 56.6 \\
\hline Oil Z2 @ $100^{\circ} \mathrm{C}(60 \mathrm{mins})$ & 0.14 & 56.6 \\
\hline Oil Z2 @ $100^{\circ} \mathrm{C}(180 \mathrm{mins})$ & 0.123 & 56.6 \\
\hline
\end{tabular}

Table 1: Summary of values of $f_{0}$ and $\sigma$ for the various oils plotted in Figure 1

Figure 1 also shows that there is a range of $X$ values. For example, at $\lambda=1, X$ is in the range $0.3-0.5$ with an average value of approximately 0.4 . It is convenient to replot the data of Figure 1 against $1 / \lambda$, and this is done in Figure 2.

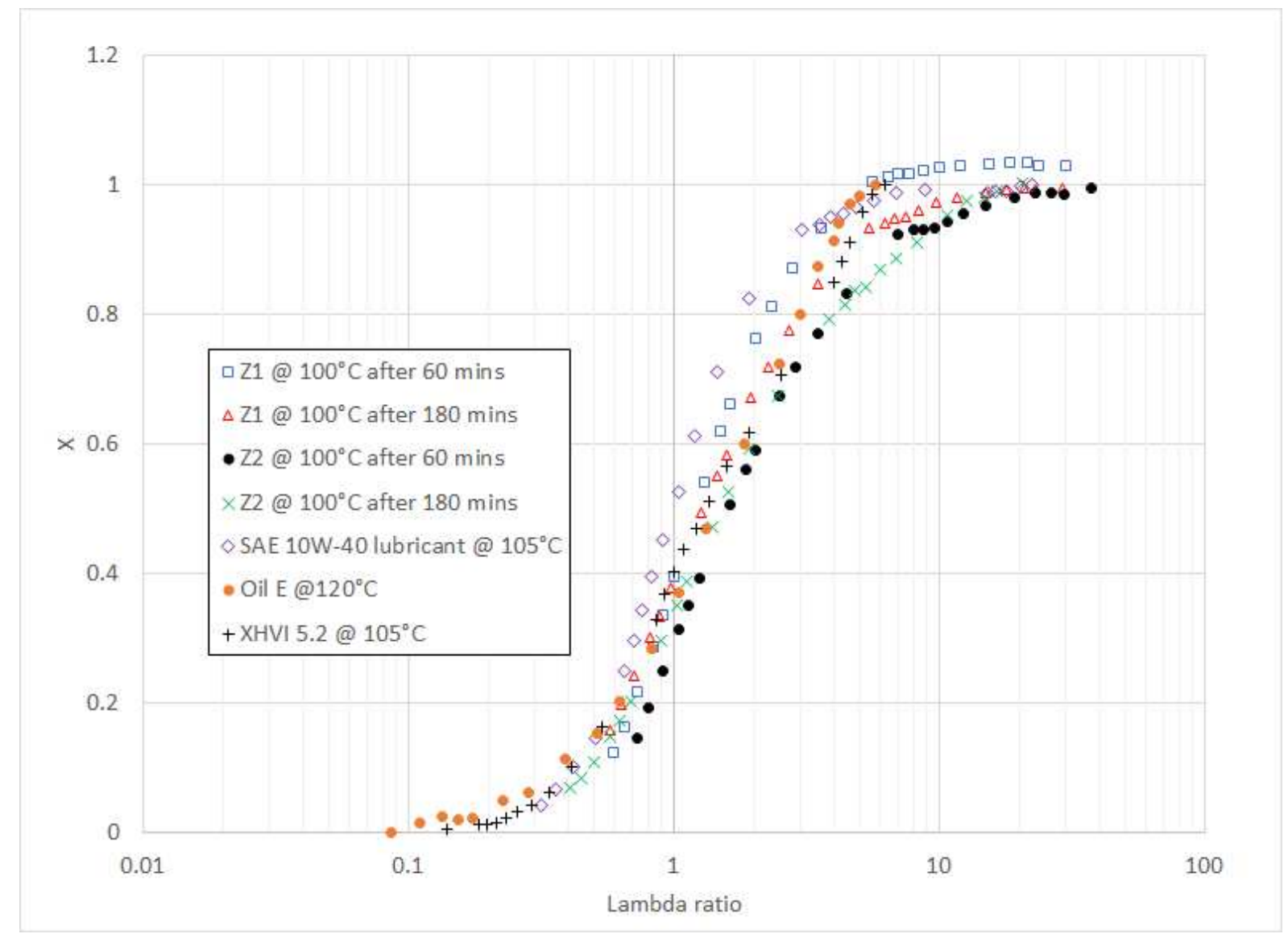

Figure 2: Replot of data from Figure 1 with horizontal axis now being $1 / \lambda$

The reason for replotting the data against $1 / \lambda$ is that it is now clear that the "universal curve" takes the form of a logistic curve $[17,18]$, also commonly referred to as a "Sigmoid" or "S-curve". A 
comprehensive review of Logistic curves and their widespread appearance in the natural and modern world can be found in a recent book by Smil [19]. A generalized form of a logistic curve that is often used is:

$$
S(x)=\frac{1}{(1+\exp (-k x))^{a}}
$$

Where $k$ and $a$ are fitting parameters. We note that in Figure 2 , the $x$-axis is actually $\log _{10}(1 / \lambda)$. If Figure 2 were replotted with the horizontal axis being $\log _{\mathrm{e}}(1 / \lambda)$, the fundamental shape of the curves would not change (although the horizontal axis would have a different scale) and if $\log _{e}(1 / \lambda)$ is used in equation (3), this would lead to the following function that could be used to fit the data in Figure 2:

$$
S(\lambda)=\frac{1}{\left(1+\lambda^{k}\right)^{a}}
$$

Figure 3 shows a comparison of the measured friction data and a best fit, using the form of $S(\lambda)$ above (with $k=1.517$ and $a=1.327$ ). The best fit values of $k$ and a were found by minimizing the sum of the normalized squares of the differences between the fitted curve and the data points, using Excel's Solver function. (To a good approximation, the following parameters for $\mathrm{k}$ and a give a good fit to the data: $\mathrm{k} \approx \frac{3}{2}$ and $\mathrm{a} \approx \frac{4}{3}$ ).

A function of similar form has previously been proposed by Olver and Spikes in 1998 [20] where, in equation (21) of that paper, the equation $X=(1+\lambda)^{-m}$ was suggested for the proportion of mixed/boundary friction, with a value of $m$ of 2 , which is a special case of equation (4) above, when $k=1$. 


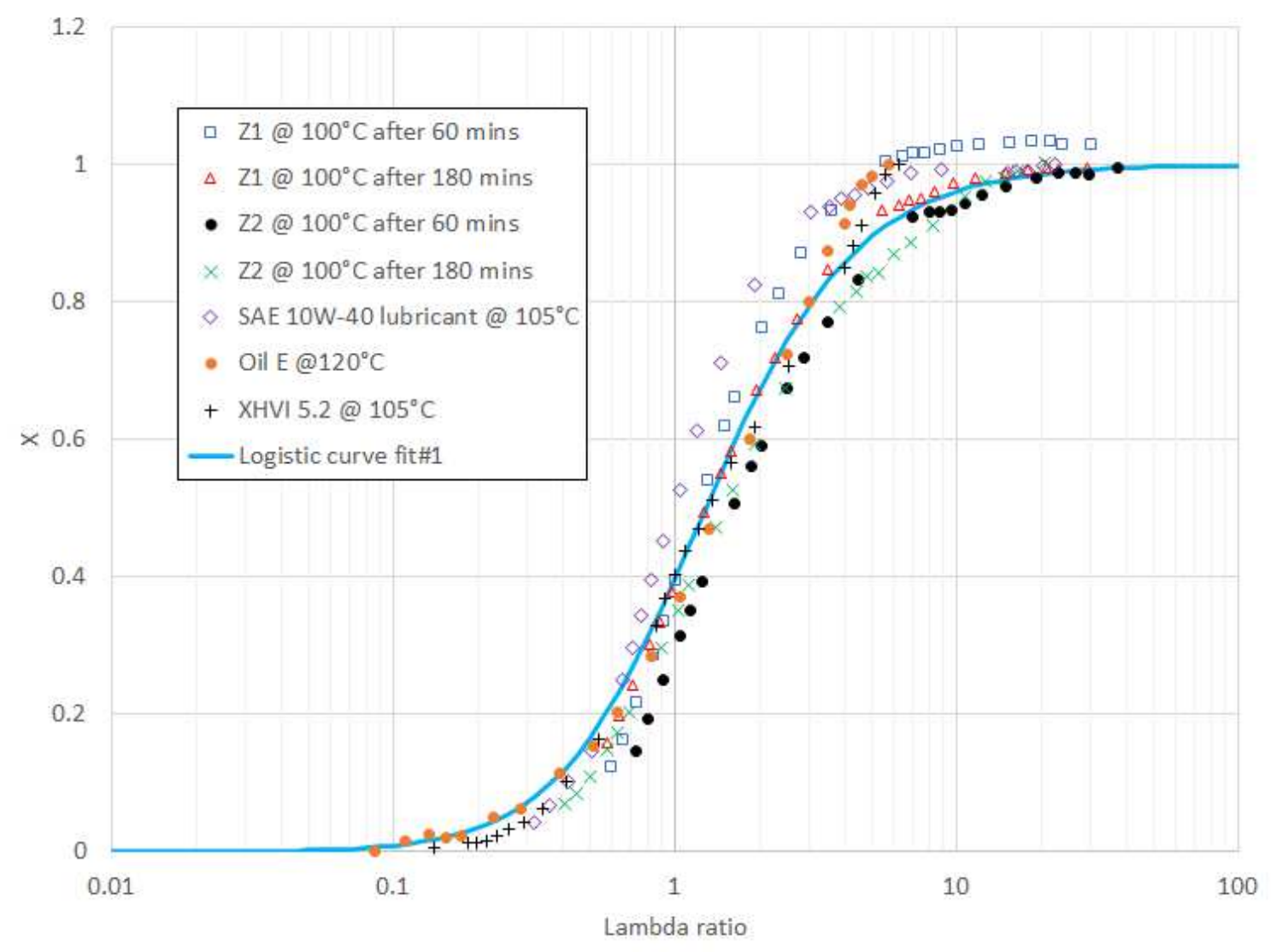

Figure 3: Replot of Figure 2 that includes a curve fit using $S(\lambda)$ above, with $k=1.517$ and $a=1.327$

The use of a logistic function to model mixed/boundary friction has the following advantages:

- There is no artificial cut-off. In many models of mixed/boundary friction, there is an assumption that the mixed/boundary friction is zero if $\lambda$ is greater than a certain value (usually values of 3 or 4 are assumed).

- The gradient of $X$ is zero at both low and high $\lambda$ values.

- $X$ is the proportion of mixed/boundary friction, and clearly increases with $1 / \lambda$ (or alternatively, decreases as $\lambda$ increases). In addition, it is known that the real contact area also increases with $1 / \lambda$ too. Logistics curves often arise from well-studied generalized differential equations, and it is suggested that such an approach may be useful for the prediction of $X$ (and/or prediction of the real contact area).

Many well-known asperity contact models predict the load carried by the asperities, and if this is divided by the total load, a value of $X$ can be obtained which can be compared with that of the "universal curve" reported above.

\section{Comparison with Previous Mixed/Boundary Friction Models}

Greenwood and Williamson [3] calculated the load supported by the asperities, $W(\lambda)$, and the real area of contact, when a rough surface contacted a flat surface. The expression for the load supported by the asperities was of the form: $W(\lambda)=A F(\lambda)$, where $A$ is a pre-factor that depends on statistical details of rough surface, and also includes materials properties (such as Young's modulus) and $F(\lambda)$ is a function that depends only on the $\lambda$ ratio. The pre-factor $A$ is equal to $W(0) / F(0)$, so that $W(\lambda) / W(0)$ 
$=F(\lambda) / F(0)$. It is assumed that $W(\lambda) / W(0)$ is the same as the quantity $X$ reported in the previous section. Two expressions were reported in [3], one for the case in which the peak heights of the rough surface were distributed exponentially, and the other for the case in which the peak heights had a Gaussian distribution.

When the probability distribution function of peak heights was assumed to be an exponential distribution, Greenwood and Williamson [3] reported that:

$$
X=\exp (-c \lambda)
$$

On the other hand, if it was assumed that the probability distribution function of peak heights was a Gaussian distribution, then:

$$
X=\frac{F_{3 / 2}(c \lambda)}{F_{3 / 2}(0)}
$$

Where:

$$
F_{n}(u)=\frac{1}{\sqrt{2 \pi}} \int_{u}^{\infty}(s-u)^{n} \cdot \exp \left(-\frac{s^{2}}{2}\right) \cdot d s
$$

Note that in equations (4) and (5), a factor c has been introduced, to allow for the possibility that the standard deviation of the probability distribution function for peak heights could be different from the standard deviation of the probability distribution function of the overall surface. In practice, however, most engineers and tribologists, in the absence of any further information, would assume that $c=1$.

A later model, discussed by Greenwood and Tripp [4], assumed both surfaces were rough, and the following expression was derived, which is still in widespread use today:

$$
X=\frac{F_{5 / 2}(c \lambda)}{F_{5 / 2}(0)}
$$

The $F_{3 / 2}(x)$ and $F_{5 / 2}(x)$ are quite complex functions, although recently an exact expression for these functions has been reported by Jedynak et al [21].

$$
F_{n}(\lambda)=\frac{\Gamma(n+1)}{\sqrt{2 \pi}} \cdot \exp \left(-\frac{\lambda^{2}}{4}\right) \cdot U\left(n+\frac{1}{2}, \lambda\right)
$$

Where $\Gamma(\mathrm{x})$ is the Gamma function, and $\mathrm{U}(\mathrm{m}, \mathrm{x})$ is the parabolic cylinder function.

In practice, although exact equations are available for $F_{n}(\lambda)$, these are also not that straightforward to use easily and most engineers would generally use tabulated values of these functions, at specific values of $\lambda$, and simply interpolate for intermediate $\lambda$ values (tabulated values of these functions can be found in the paper by Greenwood and Tripp [4]). 
As discussed in the previous section, a comparison of measured friction coefficients (from the Mini Traction Machine) and lambda ratio inferred the following relationship:

$$
X=\frac{1}{\left(1+\lambda^{k}\right)^{a}}
$$

With $\mathrm{k}=1.517$ and $\mathrm{a}=1.327$.

As mentioned earlier, a similar type of expression was reported by Olver and Spikes in 1998 [20]:

$$
X=\frac{1}{(1+\lambda)^{m}}
$$

Where the value of $m$ was reported to be equal to 2 .

Other expressions for $\mathrm{X}$ have also been reported, although often these apply to a restricted range of $\lambda$ values. Examples include the equation below from Castro and Seabra [22]:

$$
X=\operatorname{Max}\left(\left(1-0.84 \lambda^{0.23}\right), 0\right)
$$

An expression reported by Zhu et al [23] is:

$$
X=1-\frac{1.21 \lambda^{0.64}}{1+0.37 \lambda^{1.26}}
$$

Also recently, Sander et al [24] has approximated $X$, for $\lambda<4$ by:

$$
X=\left(1-\frac{\lambda}{4}\right)^{6.804}
$$

For $\lambda>4$, Sander et al [24] simply assumed that $X$ was zero. In fact, the above expression is a good approximation for $F_{5 / 2}(\lambda) / F_{5 / 2}(0)$.

A linear approximation for calculating the friction coefficient in automotive valve trains was proposed by Coy [25], and this would result in the following equation for $X$ (for $\lambda<3$ ):

$$
X=\left(1-\frac{\lambda}{3}\right)
$$

In the above expression, if $\lambda>3, x=0$.

A comparison of the Logistic curve fit, the Greenwood and Williamson exponential fit [3], the Greenwood and Tripp fit [4], a simple linear fit [25], and the Olver and Spikes fit [20], with the MTM experimental data is shown in Figure 4 below. 
Clearly, the Logistics curve fit shown in Figure 4 is a good fit to experimental friction data from the Mini Traction Machine. It can also be seen that that many of the surface contact models used in mixed/boundary friction prediction lead to significant underestimates of mixed/boundary friction. For example, the widely used Greenwood \& Tripp model [4] predicts a value for $X$ of about 0.13 when $\lambda=$ 1 , compared to a value of 0.399 from the Logistic curve fit. At a value of $\lambda=0.1$ the difference is less (with the logistics curve fit giving a normalized friction of 0.96 and the Greenwood Williamson/Greenwood Tripp models giving a value of approximately 0.84 ).

Of the simple models in the published literature, the one that comes closest to the Logistics curve fit is the exponential function, $X=\exp (-\lambda)$ (which predicts a value of $X$ of 0.368 at $\lambda=1$ ). The linear curve can clearly be seen to overestimate $X$ in the mixed lubrication regime.

Table 2 lists the value of $X$, from the different approximations, for selected values of $\lambda$.

\begin{tabular}{|l|c|c|c|c|c|}
\hline$\lambda$ & \multicolumn{5}{|c|}{$\mathrm{X}$} \\
\hline & $\begin{array}{c}\text { Logistic curve } \\
\text { (eq. 10) } \\
\mathrm{k}=1.517, \mathrm{a}=1.327\end{array}$ & $\exp (-\lambda)$ & $\begin{array}{c}\text { Linear fit } \\
\text { (eq. 15) }\end{array}$ & $\begin{array}{c}\text { Olver \& Spikes [20] } \\
\text { (eq. 11) }\end{array}$ & $\begin{array}{c}\text { Greenwood- } \\
\text { Tripp [4] }\end{array}$ \\
\hline 3 & 0.0872 & 0.0498 & 0 & 0.0625 & 0.00028 \\
\hline 2 & 0.167 & 0.135 & 0.333 & 0.111 & 0.0088 \\
\hline 1 & 0.399 & 0.368 & 0.667 & 0.25 & 0.131 \\
\hline 0.5 & 0.672 & 0.607 & 0.833 & 0.444 & 0.390 \\
\hline 0.2 & 0.895 & 0.819 & 0.933 & 0.694 & 0.698 \\
\hline 0.1 & 0.961 & 0.905 & 0.967 & 0.826 & 0.838 \\
\hline
\end{tabular}

Table 2: Predictions of $X$ for selected $\lambda$ values for the different asperity models 


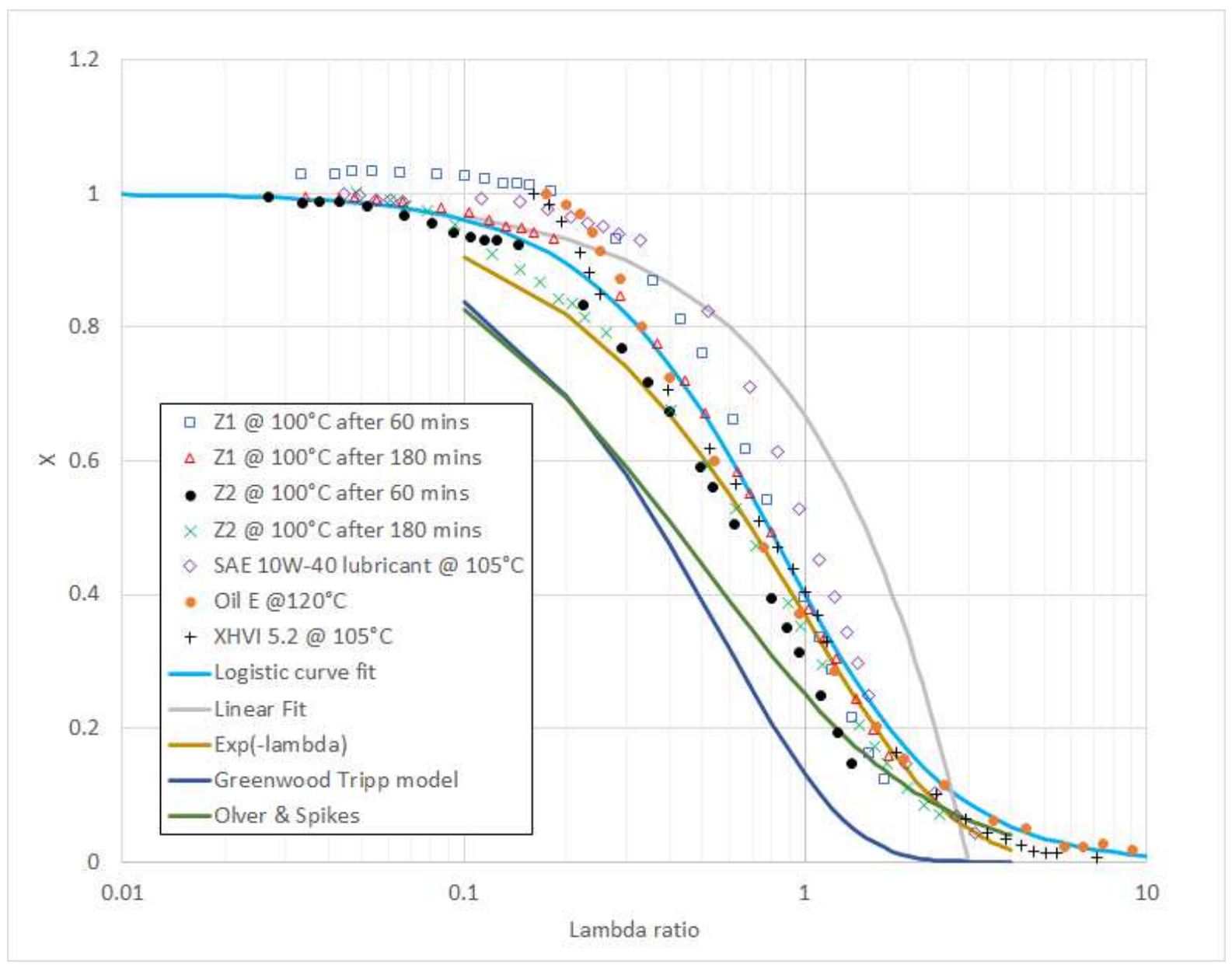

Figure 4: Comparison of various approximations of $X$ versus $\lambda$

The conclusion reached here, that the Greenwood \& Tripp model leads to significant underestimates of friction in the mixed/boundary lubrication regime, agrees with recent work by Leighton et al [26]. Those authors used a generalized function $F_{n L}(\lambda)$ defined by:

$$
F_{n L}(\lambda)=\int_{\lambda}^{\infty}(s-\lambda)^{n} \cdot \phi^{*}(s) \cdot d s
$$

Where $\phi^{*}(\mathrm{~s})$ is the probability distribution function for the peak height distribution of the surfaces. If the peak height distribution is assumed to be Gaussian, this is equivalent to the expression given in equation (7). However, Leighton et al [26] used the measured probability distribution of a real surface, and found that the absolute values of both $F_{n L}(\lambda)$ and $F_{n L}(\lambda) / F_{n L}(0)$ were substantially greater than the equivalent expressions based on a Gaussian probability distribution function. For example, as mentioned above, $F_{5 / 2}(\lambda) / F_{5 / 2}(0)$ is only around 0.13 when $\lambda=1$, whereas the logistics curve fit gave a value of $X$ of approximately 0.399 for $\lambda=1$. The value of $F_{n L}(\lambda) / F_{n L}(0)$ is approximately 0.22 when $\lambda=1$ (see Figure 6 in reference [26]), which is greater than for the Gaussian approximation, but still less than predicted from the logistics curve fit to the "universal" curve. 


\section{Asymptotic Limits of the Logistics Curve Fit}

As discussed before the Logistics curve fit is:

$$
X=\frac{1}{\left(1+\lambda^{k}\right)^{a}}
$$

It is worth looking at the asymptotic limits of the above expression for the limits $\lambda \rightarrow 0$ and $\lambda \rightarrow \infty$.

For large values of $\lambda$, it is clear that $X \approx \lambda^{\text {-ak }}$. This should be valid for $\lambda>2$

On the other hand, for small values of $\lambda$

$$
X \approx 1-a \lambda^{k}
$$

The above expression should be valid for $\lambda<0.3$.

A comparison of these asymptotic limits with the full logistics curve is shown in Figure 5.

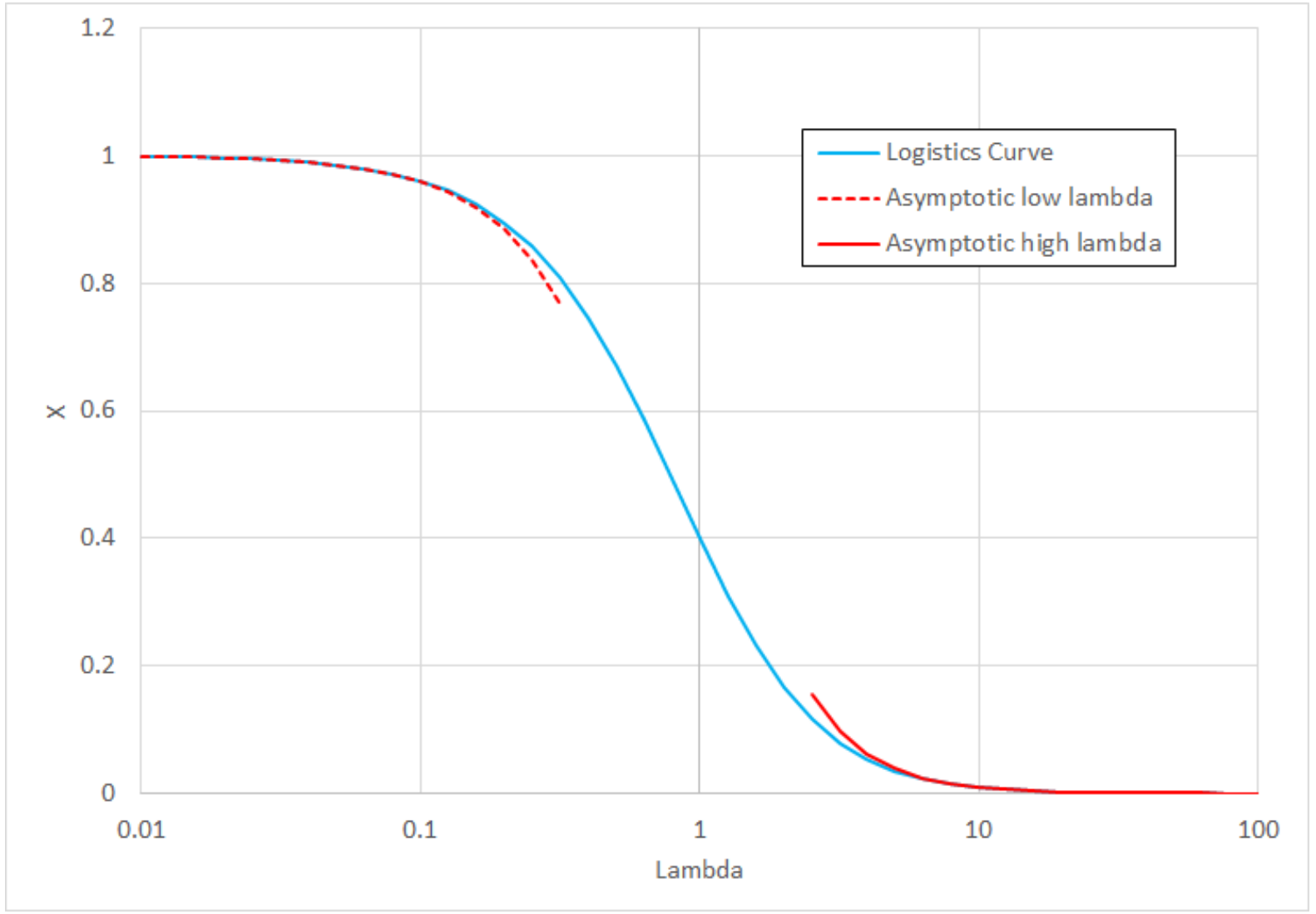

Figure 5: Comparison of asymptotic limit expressions (for low and high $\lambda$ ) with full logistics curve (in this case $\mathrm{k}=1.517$ and $\mathrm{a}=1.327$ ) 
In the high $\lambda$ limit, $X \approx \lambda^{\text {-ak }}$ which for the values of $k$ and a found for the best fit curve, would lead to $X$ $\propto \lambda^{-2.01}$. For hydrodynamic lubrication, it is often stated [40] that $\lambda \propto(\eta \mathrm{U} / \mathrm{W})^{0.5}$ (where $\eta=$ dynamic viscosity (Pa.s), $U=$ sliding speed $(\mathrm{m} / \mathrm{s})$ and $W=\operatorname{load}(\mathrm{N}))$. Therefore, with these assumptions, for high $\lambda, X \approx W$, i.e. the proportion of mixed/boundary lubrication at high $\lambda$ would be roughly proportional to applied load. A previous expression for $X$, due to Olver and Spikes [20] had the form of equation (17) with $k=1$ and $a=2$. This curve would have an asymptotic limit at high $\lambda(\lambda>2)$ such that $X \propto \lambda^{-2}$. With the logistic curve found here, if it is assumed that $k \approx \frac{3}{2}$ and $a \approx \frac{4}{3}$ then $a k=2$ and the relationship $\mathrm{X} \propto \lambda^{-2}$ would hold exactly, for large $\lambda$.

Although the above discussion has focussed on $X$ being the proportion of the load supported by the asperities, it is worth pointing out it could also be viewed as being the proportion of real contact area to the apparent contact area. In Greenwood \& Tripp's original model [4], the equation for $W(\lambda) / W(0)$ is given by equation (8). In that work, it was also reported that the real contact area, $A(\lambda)$ was given by:

$$
\frac{A(\lambda)}{A(0)}=\frac{F_{2}(\lambda)}{F_{2}(0)}
$$

A numerical comparison has been carried out by the authors and it was found that the functions $F_{5 / 2}(\lambda) / F_{5 / 2}(0)$ and $F_{2}(\lambda) / F_{2}(0)$ are essentially identical, and so $X$ can also be considered to be $A(\lambda) / A(0)$, and as such the asymptotic analysis above would suggest that the real contact area should be roughly proportional to load, at high $\lambda$ values, as expected. (The same holds true for Greenwood \& Williamson's model [3], where, for a Gaussian distribution of surface roughness, $W(\lambda) / W(0)=$ $F_{3 / 2}(\lambda) / F_{3 / 2}(0)$ and $A(\lambda) / A(0)=F_{1}(\lambda) / F_{1}(0)$. A numerical comparison of these functions shows that $W(\lambda) / W(0)$ is virtually identical to $A(\lambda) / A(0))$.

\section{A New Approach to Mixed/Boundary Friction Calculation}

The fact that the Universal curve proposed by Dawczyk et al [13] is well fitted by a Logistics curve suggests a new approach for predicting the proportion of mixed/boundary friction, $X$. A good summary of the type of differential equations that lead to Logistics curves has been published by Tsoularis [27]. Of the various differential equations discussed, the one proposed by Blumberg [28] leads to a differential equation of the following form:

$$
\frac{d X}{d \lambda}=-\alpha X^{\beta}(1-X)^{\gamma}
$$

Where $\alpha, \beta, \gamma$ are constants.

The above equation differs slightly from that reported by Blumberg [28] since a negative sign has been used on the right-hand side of the equation. This is because $X$ decays with $\lambda$. 
The above equation effectively states that the rate of change of $X$ tends to zero when (1) there are very few contacts, which tends to occur at high $\lambda$ and (2) when $\lambda$ is small, since then the amount of mixed/boundary friction cannot change very much as it is already very close to 1 .

No assumption has been made in the above equation as to whether contact conditions are plastic or elastic.

A general analytical solution of equation (20) is not readily available, but the above equation can be solved numerically.

By assuming that $X=0.998772$ at $\lambda=0.01$ (the value predicted by the logistic curve fit), a numerical solution to the above equation was found that fits the logistic curve fit found earlier, with the following parameters:

$\alpha=1.88, \beta=1.49$ and $\gamma=0.35$

In any given contact, these constants may depend on the precise details of the rough surfaces, the lubricant additives and the material properties of those surfaces. Further work is needed to better understand the dependence of $\alpha, \beta$ and $\gamma$ on these parameters.

A comparison of the numerical solution of equation (18) with the logistics curve fit found earlier is shown in Figure 6 below.

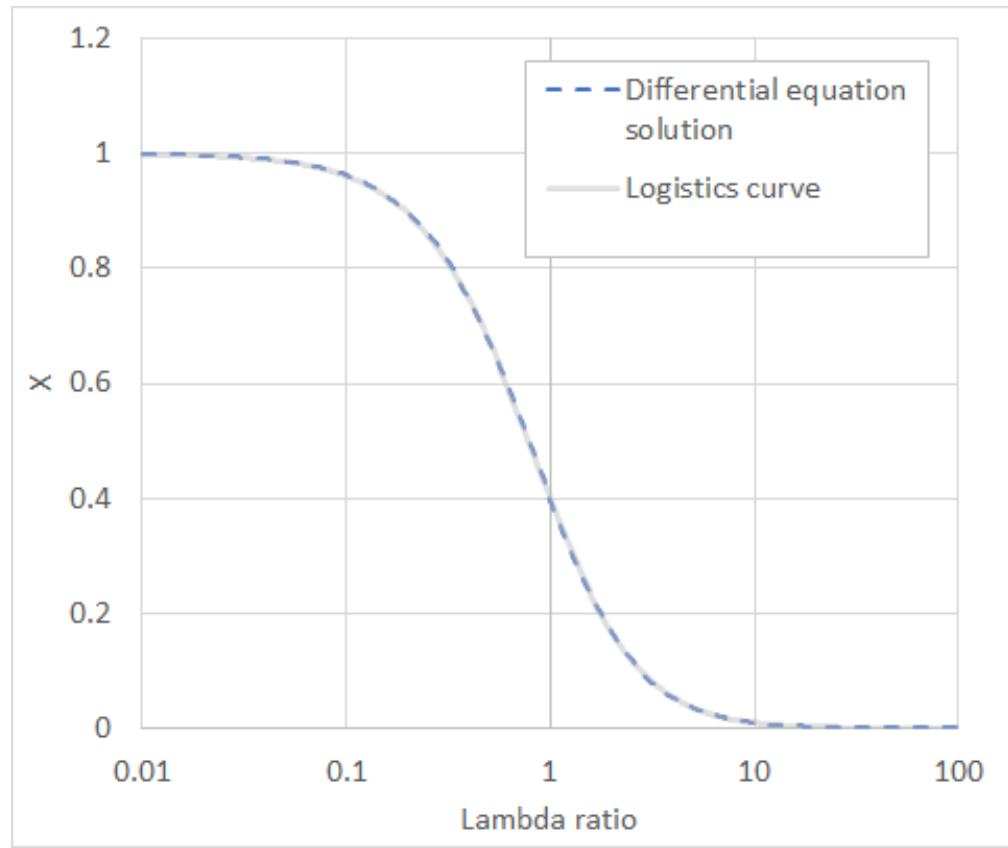

Figure 6: Comparison of solution of equation (20) (with $\alpha=1.88, \beta=1.49$ and $\gamma=0.35$ ) and the logistics curve fit (equation (10), with $\mathrm{k}=1.517$ and $\mathrm{a}=1.327$ )

Since $X$ can be considered to be the fractional real contact area, the differential equation above could be used as a "global" approach for estimating the real area of contact of two lubricated surfaces which could complement existing local approaches that have previously been considered (where detailed elastic or plastic deformation models have been used, together with the statistics of the contacting surfaces, to estimate real contact areas). 


\section{Application to Engineering Problems}

The friction force due to mixed/boundary friction, $\mathrm{F}_{\mathrm{B}}$, for a particular lubricant, would simply be given by

$$
F_{B}=f_{o} \cdot X \cdot W
$$

where $f_{0}$ is the friction coefficient for the lubricant (at $\left.\lambda=0\right), X$ is the proportion of load carried by the asperities, and $W$ is the total load acting on the contact. In this paper $X$ would be calculated using the logistics curve fit (for the appropriate value of $\lambda$ ).

The power loss due to mixed/boundary friction, $\mathrm{P}_{\mathrm{B}}$, would likewise be given by

$$
P_{B}=f_{o} \cdot X \cdot W \cdot U
$$

where $U$ is the relative (sliding) speed of the moving surfaces.

For low-speed contacts, it is possible to have relatively high mixed/boundary friction forces, but relatively low power losses due to mixed/boundary lubrication. This happens, for example, in the piston assembly of an internal combustion engine, where oil films are thin close to top and bottom dead centre positions, but piston speeds are also very low (at these positions) leading to the collapse of hydrodynamic lubricating films.

As a specific example, consider the use of the work described here to predict mixed/boundary friction losses in the valve train of an internal combustion engine. The valve train is often considered, by many researchers, to be the engine component which is the major source of mixed/boundary lubrication in an engine [29-33]. Numerous researchers have measured (or predicted) valve train friction [34-39] and found it can be as high as $40 \%$ of total engine friction at low engine speeds for a fully warmed up engine, and this would primarily all be mixed/boundary friction. The proportion of valve train friction in most modern engines is likely to be lower (due to the use of lower weight materials, and softer springs, and also because many new vehicles use stop-start systems that switch the engine off when the vehicle is stationary so that there is much less engine idling than previously), but it is still anticipated that, for passenger cars, the valve train will be the main source of mixed/boundary lubrication in an engine.

Figure 7 shows the predicted valve train friction torque for a typical bucket type overhead camshaft for different mixed/boundary friction models (Logistics curve, Greenwood Tripp model, and linear friction model [25]). Clearly, the predicted friction depends on many specific details of the cam profile and spring properties. For these simulations, the cam base circle was assumed to have a radius of 17.5 $\mathrm{mm}$, the maximum valve lift was approximately $9 \mathrm{~mm}$, the mass of the moving components in the valve train was assumed to be $0.17 \mathrm{~kg}$, the preload was assumed to be $300 \mathrm{~N}$, and the spring constant was assumed to be $40 \mathrm{~N} / \mathrm{mm}$. For the lubricant, the pressure viscosity coefficient was assumed to be $12 \mathrm{GPa}^{-1}$, and the lubricant viscosity was assumed to be $10 \mathrm{mPa}$.s, these values being typical of lubricants used at $100^{\circ} \mathrm{C}$. It was also assumed that there were 4 valves per cylinder, so 16 valves in total. The oil film thickness between cam and tappet was calculated using standard elastohydrodynamic line contact equations [40]. Figure 7(a) shows that the friction torque predicted from the Greenwood Tripp model is substantially lower than that predicted using the logistics curve fit proposed here. Typically, the value predicted by the Greenwood Tripp model is only about $70-80 \%$ of the values predicted using the logistics curve fit. Use of a linear fit (where $X=1-\lambda / 3$ for $\lambda<3$ ) 
results in slightly higher predicted valve torque. However, the linear fit does not agree well with experimental friction measurements from the MTM machine. At the lowest speeds, predicted valve train torques are close to $2 \mathrm{Nm}$ (when $\sigma$, is assumed to be $0.2 \mu \mathrm{m}$ ) which is equal to a friction mean effective pressure (FMEP) of $13 \mathrm{kPa}$, and is close to measured values (although this will vary from engine to engine, and with operating conditions, and does not include fluid film friction).

For bucket tappet valve trains, the combination of a rotating cam contacting a tappet which also rotates, generally results in quite smooth surfaces, so a typical RMS roughness would be $0.2 \mu \mathrm{m}$. If a value of $0.0567 \mu \mathrm{m}$ was used (which is the RMS roughness of surfaces covered in ZDDP films, according to Dawczyk et al [13]), lower values of friction torque would be predicted (since $\lambda$ values would be higher), and the predicted valve train friction torque for this situation is shown in Figure $7(b) . X$ was predicted using the logistics curve fit and it was assumed that $f_{\circ}$ was 0.12 .

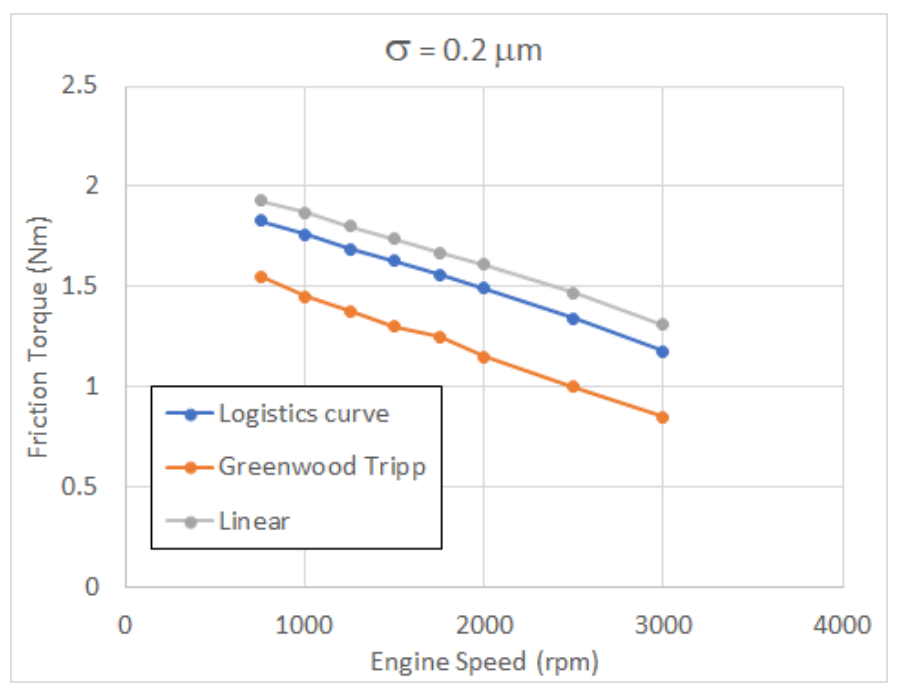

Figure 7(a)

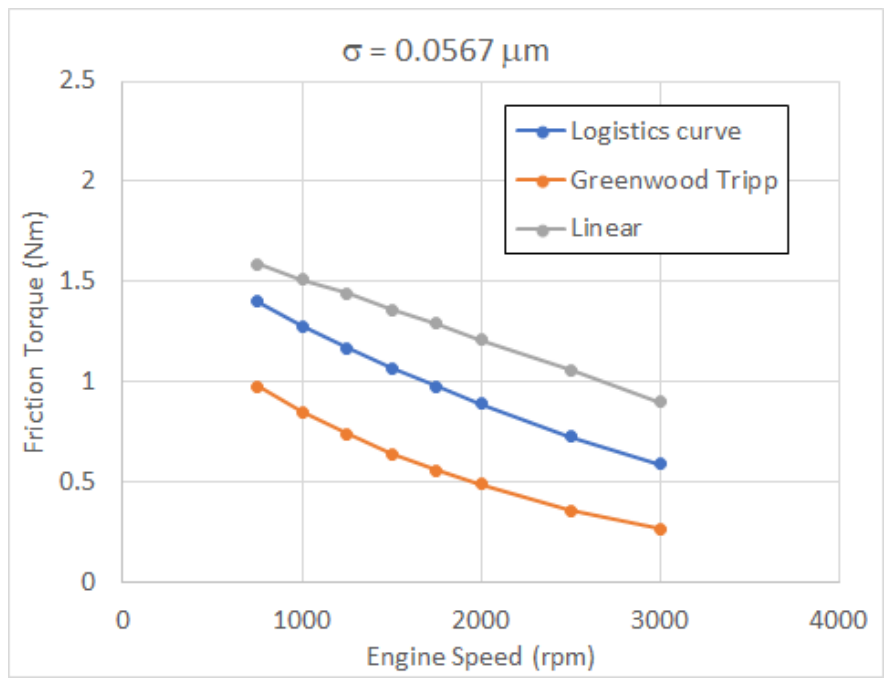

Figure $7(b)$

Figure $7(\mathrm{a})$ and $7(\mathrm{~b})$ : Predicted valve train friction torque $(\mathrm{Nm})$ versus engine speed $(\mathrm{rpm})$ for different mixed/boundary lubrication models. Figure 7 (a) shows results when the combined surface roughness is assumed to be $0.2 \mu \mathrm{m}$, and Friction 7 (b) shows results for a combined surface roughness of $0.0567 \mu \mathrm{m}$ 


\section{Discussion}

As improvements in energy efficiency increase in importance, the move to lower viscosity lubricants is likely to continue, and this will lead to increased mixed/boundary friction in machines. Accurate prediction of the proportion of mixed/boundary friction will, therefore, become increasingly important, both for friction and wear.

The important work of Dawczyk et al [13] has shown that both ZDDP containing oils, and base oils, can be plotted on a "universal" curve of $X$ versus $\lambda$ (where $X$ is the proportion of mixed/boundary lubrication) provided friction data (in this case from the Mini Traction Machine) is normalized to its value at zero film thickness, and the correct surface roughness parameters are used. In this paper, additional friction data has been analysed and it has been confirmed that this additional data also fits onto the same "universal" curve.

A good fit to the "universal" curve has been found with a relatively simple logistics curve. A comparison of the logistics curve fit with other commonly used equations for the proportion of mixed/boundary lubrication has found that some well-known (and well used) expressions (such as that due to Greenwood and Tripp [4]) lead to significant underestimates of the amount of mixed/boundary lubrication. This implies that using such models to estimate mixed/boundary friction in engineering contacts such as the valve train, may lead to significant errors and this has been demonstrated for that component using a specific case to compare the predicted mixed/boundary friction losses. Further work would be needed for surfaces that have deliberate texture, or for surfaces (such as piston liners) that contain deep honing grooves. It is possible that such surfaces may well have a "universal" curve that is different from the one found by Dawczyk et al [13].

Since Logistics curves arise from growth and decay processes, it is natural to ask whether the type of differential equations that arise in growth/decay studies can be applied to lubricated contacts. A generalized differential equation (due to Blumberg [28]) has been used to show that this approach can lead to a logistics curve that is a good fit to the "universal" curve found by the MTM measurements of Dawczyk et al [13]. No assumption has been made in writing down such a differential equation as to whether contact is elastic or plastic. Previous approaches to predicting the amount of mixed/boundary lubrication (or real contact area) have generally started with models that use elastic or plastic asperity deformation models, together with detailed statistical models of surfaces, to "buildup" the contact area, and/or to predict the proportion of load carried by asperities. As opposed to these "local" models, a "global" differential equation based on growth/decay processes could complement these earlier approaches and provide new insights.

This work has focussed on ZDDP containing lubricants and/or base oils. Further work is needed to study whether friction modified lubricants can be treated in the same fashion. This is because friction modifiers generally do not form thick tribo-film deposits. In general, it is thought friction modifier films at surfaces are only a few molecular layers thick. Therefore, friction modifier additives will not form films that are thick enough to affect the $\lambda$ ratio. However, they are known to significantly reduce the friction coefficient by their influence on the shear strength at the contact. Williams [41] has suggested that such friction modifier tribo-films can alter the relationship between stress and pressure, which can directly impact how the friction coefficient would vary with $\lambda$, and inclusion of such effects may be needed for a better understanding of the friction curve when lubricants with friction modifier additives are used. 


\section{Conclusions}

It has been shown that the "universal" normalized friction curve, for both ZDDP containing oils, and base oils, originally proposed by Dawczyk et al [13] can be fitted very well to MTM friction data from other researchers for different lubricants and can be conveniently approximated by a generalized

logistics curve. The curve found $X=\left(1+\lambda^{k}\right)^{-a}$ (with $k \approx \frac{3}{2}$ and $a \approx \frac{4}{3}$ ) provides a modelling tool which is much more straightforward (and accurate) for engineers to use for the estimation of mixed/boundary friction than other equations previously reported in the literature.

The insight that a logistics curve can fit the normalized friction in the mixed/boundary lubrication regime also suggests that a "global" approach to predicting the proportion of mixed/boundary lubrication is possible, in which no assumptions are made as to whether contact is plastic or elastic. A simple numerical solution of a generalized logistics differential equation for the proportion of mixed/boundary lubrication found a good fit for the logistics curve fit (which in turn was a good fit to the normalized "universal" friction curve, which seems to fit experimental data rather well). It is also expected that such an approach would be useful for predicting the real contact area in lubricated contacts.

The novelty of this work can be summarized as follows:

- The "universal" curve proposed by Dawczyk et al [13] for ZDDP containing oils, and base oils, has been found to apply to other MTM data on different oils from other research groups.

- A simple comparison of the "universal" curve with widely used asperity contact model predictions suggest that commonly used models (such as that due to Greenwood and Tripp [4]) significantly underestimate mixed/boundary friction, particularly in the important range $1<\lambda<3$.

- A generalized logistics curve has been found to fit the universal curve rather well, and is a simple well-behaved function that can be used to accurately predict the proportion of mixed/boundary lubrication as $\lambda$ varies.

- Logistics curve usually arise from growth/decay processes that are described by well-studied differential equations. The fact that a logistics curve is a good fit for the universal curve discussed here suggests that a differential equation could be used to predict the variation of the proportion of mixed/boundary lubrication with $\lambda$, which makes no assumptions about the deformation mode of the asperities. This could complement existing "local" approaches, where the proportion of mixed/boundary lubrication is predicted using "local" highly specific models for asperity deformation and surface topography.

\section{Acknowledgments}

The authors would like to thank Professor Hugh Spikes (Imperial College) for useful feedback on an early draft of the paper and for providing original friction data for oils Z1 and Z2 from reference [13] which greatly aided analysis work in this paper. The authors would also like to gratefully acknowledge receipt of a research grant from TTRF that funded this work.

\section{Author Contributions}

All authors contributed to the study conception and design. The first draft of the manuscript was written by Robert lan Taylor and all authors commented on previous versions of the manuscript. All authors read and approved the final manuscript. 


\section{References}

1. J.F. Archard, "Elastic Deformation and the Laws of Friction", P. Roy. Soc. Lond. A Mat., 243, pp 190-205, 1957 (https://doi.org/10.1098/rspa.1957.0214)

2. J.F. Archard, "Single Contacts and Multiple Encounters", Journal of Applied Physics, 32, 1420, 1961 (https://doi.org/10.1063/1.1728372)

3. J.A. Greenwood \& J.B.P. Williamson, "Contact of Nominally Flat Surfaces", P. Roy. Soc. Lond. A Mat., 295, pp 300-319, 1966 (https://doi.org/10.1098/rspa.1966.0242)

4. J.A. Greenwood \& J.H. Tripp, "The Contact of Two Nominally Flat Rough Surfaces", Proc. Instn. $\begin{array}{lllll}\text { Mech. } & \text { Engrs., } & \text { Vol. } & 185, & \text { 625-633, }\end{array}$ (https://doi.org/10.1243\%2FPIME PROC 1970185069 02)

5. A.W. Bush, R.D. Gibson \& T.R. Thomas, "The Elastic Contact of a Rough Surface", Wear, 35, pp 87-111, 1975 (https://doi.org/10.1016/0043-1648(75)90145-3)

6. A. Majumdar \& B. Bhushan, "Fractal Model of Elastic-Plastic Contact Between Rough Surfaces", J. Tribol. (ASME), 113, pp 1-11, 1991 (https://doi.org/10.1115/1.2920588)

7. B.N.J. Persson, "Elastoplastic Contact Between Randomly Rough Surfaces", Physical Review $\begin{array}{lllll}\text { Letters, Vol. } & \text { 87, No. 11, } & 116101\end{array}$ (https://link.aps.org/doi/10.1103/PhysRevLett.87.116101)

8. J.R. Barber, "Multiscale Surfaces and Amontons' Law of Friction", Tribology Letters, 49, pp 539-543, 2013 (https://doi.org/10.1007/s11249-012-0094-6)

9. L. Afferante, G. Carbone \& G. Demelio, "Interacting and Coalescing Hertzian Aperities: A New Multiasperity Model", Wear, 278-279, pp 28-33, 2012 (https://doi.org/10.1016/i.wear.2011.12.013)

10. Y. Xu, R.L. Jackson \& D.B. Marghitu, "Statistical Model of Nearly Complete Elastic Rough Surface Contact", Int. J. Solids \& Structures, 51, pp 1075-1088, 2014 (https://doi.org/10.1016/j.ijsolstr.2013.12.005)

11. H. Spikes, "The History and Mechanisms of ZDDP", Tribology Letters, 17, pp 469-489, 2004 (https://doi.org/10.1023/B:TRIL.0000044495.26882.b5)

12. H. Spikes, "Friction Modifier Additives", Tribology Letters, 60, 5, 2015 (https://doi.org/10.1007/s11249-015-0589-z)

13. J. Dawczyk et al, "Film Thickness and Friction of ZDDP Tribofilms", Tribology Letters, 67, pp 33-34, 2019 (https://doi.org/10.1007/s11249-019-1148-9)

14. R.I. Taylor, "Tribology and Energy Efficiency: From Molecules to Lubricated Contacts to Complete Machines", Faraday Discussions, 156, pp 361-382, 2012 (https://doi.org/10.1039/C2FD00122E)

15. S. Gunsel, S. Korcek, M. Smeeth \& H.A. Spikes, "The Elastohydrodynamic Friction and Film Forming Properties of Lubricant Base Oils", Tribology Transactions, 42(3), pp 559-569, 1999 (https://doi.org/10.1080/10402009908982255)

16. P.W. Gold, A. Schmidt, H. Dicke, J. Loos \& C. Assman, "Viscosity-Pressure-Temperature Behaviour of Mineral and Synthetic Oils", Journal of Synthetic Lubrication, 18(1), pp 51-79, 2001 (https://doi.org/10.1002/jsl.3000180105)

17. https://en.wikipedia.org/wiki/Logistic function

18. https://en.wikipedia.org/wiki/Generalised logistic function

19. V. Smil, "Growth: From Microorganisms to Megacities", MIT Press, 2019

20. A.V. Olver \& H.A. Spikes, "Prediction of Traction in Elastohydrodynamic Lubrication", Proc. IMechE. Pt. J: Journal of Engineering Tribology, 212, pp 321-332, 1998 (https://doi.org/10.1243\%2F1350650981542137) 
21. R. Jedynak, "Exact and Approximate Solutions of the Infinite Integrals of the Asperity Height Distribution for the Greenwood-Williamson and the Greenwood-Tripp Asperity Contact $\begin{array}{lllll}\text { Models", Tribology International, 130, pp 206-215, } 2019 & \end{array}$ (https://doi.org/10.1016/i.triboint.2018.09.009)

22. J. Castro \& J. Seabra, "Coefficient of Friction in Mixed Film Lubrication: Gears versus TwinDiscs", Proc. IMechE. Pt. J: Journal of Engineering Tribology, 221, pp 399-411, 2007 (https://doi.org/10.1243\%2F13506501JET257)

23. D. Zhu and Y. Hu, "A Computer Program Package for the Prediction of EHL and Mixed Lubrication Characteristics, Friction, Subsurface Stresses and Flash Temperatures, Based on Measured 3-D Surface Roughness", Tribology Transactions, 44(3), pp 383-390, 2001 (https://doi.org/10.1080/10402000108982471)

24. D.E. Sander, H. Allmaier, H.H. Priebsch, M. Witt \& A. Skiadas, "Simulation of Journal Bearing Friction in Severe Mixed Lubrication - Validation and Effect of Surface Smoothing Due to Running-In", Tribology International 96, pp 173-183, 2016 (https://doi.org/10.1016/i.triboint.2015.12.024)

25. R.C. Coy, "Practical Applications of Lubrication Models in Engines", Tribology International, 31, pp 563-571, 1998 (https://doi.org/10.1016/S0301-679X(98)00077-2)

26. M. Leighton, N. Morris, R. Rahmani \& H. Rahnejat, "Surface Specific Asperity Model for Prediction of Friction in Boundary and Mixed Regimes of Lubrication", Meccanica, 52, pp 2133, 2017 (https://doi.org/10.1007/s11012-016-0397-z)

27. A. Tsoularis, "Analysis of Logistic Growth Models", Mathematical Biosciences, 179, pp 21-55, 2002 (https://doi.org/10.1016/S0025-5564(02)00096-2)

28. A.A. Blumberg, "Logistic Growth Rate Functions", J. Theoret. Biol., 21, pp 42-44, 1968 (https://doi.org/10.1016/0022-5193(68)90058-1)

29. J.T. Staron \& P.A. Willermet, "An Analysis of Valve Train Friction in Terms of Lubrication Principles", SAE 830165, 1983 (https://doi.org/10.4271/830165)

30. C.M. Taylor, "Valve Train - Cam and Follower: Background and Lubrication Analysis", Tribology Series, Vol. 26, pp 159-181, 1993 (published by Elsevier)

31. R.A. Mufti \& M. Priest, "Experimental and Theoretical Study of Instantaneous Engine Valve Train Friction", Journal of Tribology, Vol. 125, pp 628-637, 2003 (https://doi.org/10.1115/1.1538189)

32. C. Knauder, H. Allmaier, D.E. Sander \& T. Sams, "Investigations of the Friction Losses of Different Engine Components. Part 1: A Combined Approach for Applying SubassemblyResolved Friction Loss Analysis on a Modern Passenger-Car Diesel Engine", Lubricants, Vol. 7, Issue 5, article 7050039, 2019 (https://doi.org/10.3390/lubricants7050039)

33. R.I. Taylor, N. Morgan, R. Mainwaring \& T. Davenport, "How Much Mixed/Boundary Friction is There in an Engine - and Where Is It?", Proc. IMechE. Pt. J: Journal of Engineering Tribology, 234, pp 1563-1579, 2019 (https://doi.org/10.1177\%2F1350650119875316)

34. W.F. Ball, N.S. Jackson, A.D. Pilley \& B.C. Porter, "The Friction of a 1.6 Litre Automotive Engine - Gasoline and Diesel", SAE 860418, 1986 (https://doi.org/10.4271/860418)

35. M.L. Monaghan, "Engine Friction - A Change in Emphasis", Proc. IMechE. Pt. D: Journal of $\begin{array}{lllll}\text { Automobile } \quad \text { Engineering, } & \text { 202, } & \end{array}$ (https://doi.org/10.1243\%2FPIME PROC $1988 \quad 202 \quad 178$ 02)

36. R.I. Taylor, "Engine Friction: The Influence of Lubricant Rheology", Proc. IMechE. Pt. J: Journal $\begin{array}{lllll}\text { of } \quad \text { Engineering } & 1997\end{array}$ (https://doi.org/10.1177\%2F135065019721100306)

37. P.J. Shayler, D.K.W. Leong \& M. Murphy, "Friction Teardown Data from Motored Engine Tests on Light Duty Automotive Diesel Engines at Low Temperatures and Speeds", Proceedings of 
the 2003 Fall Technical Conference of the ASME Internal Combustion Engine Division, ICEF2003-745, 2003 (https://doi.org/10.1115/ICEF2003-0745)

38. R.A. Mufti, "Total and Component Friction in a Motored and Firing Engine", PhD Thesis, Leeds University, 2004

39. E. Lizarraga-Garcia, T. Davenport, P. Carden, A. de Vries, S. Cakebread, S. Remmert \& R. Mainwaring, "Lubricant Impact on Friction by Engine Component: A Motored Friction Tear Down Assessment of a Production 3.6L Engine", SAE 2019-01-2239, 2019 (https://doi.org/10.4271/2019-01-2239)

40. G.W. Stachowiak \& A.W. Batchelor, "Engineering Tribology", Tribology Series 24 (published by Elsevier, 1993)

41. J.A. Williams, "The Behaviour of Sliding Contacts Between Non-Conformal Rough Surfaces Protected by 'Smart' Films", Tribology Letters, 17(4), pp 765-778, 2004 (https://doi.org/10.1007/s11249-004-8085-x) 